Research Paper

\title{
Preoperative SCC-Ag and thrombocytosis as predictive markers for pelvic lymphatic metastasis of squamous cervical cancer in early FIGO stage
}

\author{
Fan $\mathrm{Xu}^{1,2^{*}}$, Yanfang $\mathrm{Li}^{3^{*}}$, Liangsheng Fan ${ }^{*}$, Jing Ma ${ }^{1,5}$, Lan $\mathrm{Yu}^{4}{ }^{4}$, Hongyan $\mathrm{Yi}^{1}{ }^{1}$, Xiaojing Chen ${ }^{1}$, Wenfei Wei ${ }^{1}$,
} Peng $\mathrm{Wu}^{6}$, Li Liang ${ }^{7}$, Huiquan $\mathrm{Hu}^{2}$, Hui Xing ${ }^{8}$, Wei Wang ${ }^{1,4} \bowtie$

1. Department of Obstetrics and Gynecology, Nanfang Hospital, Southern Medical University, Guangzhou, Guangdong 510515, PR China

2. Department of Obstetrics and Gynecology, Nanchong Central Hospital, North Sichuan Medical University, Nanchong, Sichuan 637000, PR China

3. Department of Gynecologic Oncology, Sun Yat-sen University Cancer Center; Sun Yat-sen University, Guangzhou, Guangdong 510060, PR China

4. Department of Obstetrics and Gynecology, The First Affiliated Hospital of Guangzhou Medical University, Guangzhou, Guangdong 511436, PR China

5. Department of Obstetrics and Gynecology, People's Hospital of Three Gorges University, Yichang, Hubei 443000, PR China

6. Cancer Biology Research Center, Tongji Hospital, Tongji Medical College, Huazhong University of Science and Technology, Wuhan, Hubei 430030, PR China

7. Department of Pathology, Nanfang Hospital, Southern Medical University, Guangzhou, Guangdong 510515, PR China

8. Department of Obstetrics and Gynecology, Xiangyang Central Hospital, Hubei University of Arts and Science, Xiangyang, Hubei 441021, PR China

* These authors contributed equally to this work

$\bowtie$ Corresponding author: Wei Wang, e-mail: smugowwang@126.com, Tel: +86-13508668281; Hui Xing, e-mail: huixing1969@163.com, Tel: +86-18620759633.

(C) Ivyspring International Publisher. This is an open access article distributed under the terms of the Creative Commons Attribution (CC BY-NC) license (https://creativecommons.org/licenses/by-nc/4.0/). See http://ivyspring.com/terms for full terms and conditions.

Received: 2017.11.27; Accepted: 2018.02.10; Published: 2018.04.18

\begin{abstract}
Objectives: To explore the clinical significance of squamous cell carcinoma antigen (SCC-Ag) and thrombocytosis to predict pelvic lymphatic metastasis (PLM) of squamous cervical cancer (SCC) in International Federation of Gynecology and Obstetrics (FIGO) stages IA-IIA.

Methods: A retrospective clinicopathologic review of 782 patients of a primary cohort in three Chinese hospitals from 2010 to 2015, and 407 patients of a validation cohort in another institution from 2015 to 2017. A receiver operating characteristic curve was used to determine the optimal SCC-Ag threshold to predict PLM in the groups. Univariate and multivariate logistic analyses for PLM were performed to assess differences in outcome.

Results: In the primary and validation cohort, $15.6 \%$ (122/782) and 25.3\% (103/407) patients were classified into the thrombocytosis group (platelet count $>300 \times 10^{9} / \mathrm{L}$ ), respectively. Optimal cutoff values of SCC-Ag for predicting PLM of the thrombocytosis group and the normal group were 3.26 $\mathrm{ng} / \mathrm{mL}$ (AUC 0.754; sensitivity 73.08\%; specificity 72.92\%; $\mathrm{P}=0.000$ ) and $4.58 \mathrm{ng} / \mathrm{mL}$ (AUC 0.706; sensitivity $53.26 \%$; specificity $83.98 \% ; P=0.000$ ), respectively, in the primary cohort, and $1.55 \mathrm{ng} / \mathrm{mL}$ (AUC 0.705; sensitivity 79.31\%; specificity 55.41\%; $P=0.000$ ) and $1.75 \mathrm{ng} / \mathrm{mL}$ (AUC 0.655; sensitivity 69.57\%; specificity 64.26\%; $P=0.000$ ), respectively, in the validation cohort. In multivariate logistic analysis, preoperative SCC-Ag over $3.26 \mathrm{ng} / \mathrm{mL}$ and lymphovascular space involvement were the significant predictors of PLM for SCC in FIGO stages IA-IIA.

Conclusions: Preoperative SCC-Ag alone or combined with thrombocytosis might be used as predictive markers for PLM before initial treatment in early stage SCC.
\end{abstract}

Key words: cervical cancer, pelvic lymphatic metastasis, squamous cell carcinoma antigen, thrombocytosis

\section{Introduction}

Disease status at initial diagnosis is a crucial factor for primary treatment in cervical cancer [1]. Lymphatic metastasis can mainly cause the mortality related to cervical cancer [2], and the prognosis for patients with pelvic lymphatic metastasis (PLM) positive is not favorable [3]. Clinicopathologic 
variables, including advanced FIGO stage, large tumor size, stromal invasion depth, lymphovascular space involvement, and parametrial involvement, can influence the presence of lymphatic metastasis $[4,5]$. However, none of these factors could accurately predict lymphatic metastasis before initial treatment. In this paper, we wanted to clarify the clinical usefulness of blood biomarkers to predict pelvic lymphatic metastasis (PLM) for squamous cervical cancer (SCC) in the early stage.

The clinical value of squamous cell carcinoma antigen (SCC-Ag) was considered as a serum tumor marker for SCC, which has been demonstrated in numerous studies. For example, many studies have reported that SCC-Ag can be used to monitor patients as they receive therapy and to detect early recurrence $[6,7]$. However, there is still a debate about the predictive value of pretreatment SCC-Ag for lymphatic metastasis. Gaarenstroom et al [8] reported that SCC-Ag levels were tightly related to tumor burden, but they are not reliable to identify whether the patients are at risk of lymph node metastasis.

Increasing evidence supports that thrombocytosis plays a significant role in improving cancer biology. Platelets are associated with metastasis, angiogenesis, and tumor cell proliferation $[9,10]$. For instance, Hernandez et al [11] reported that thrombocytosis is an independent indicator of poor prognosis for patients with cervical cancer. Therefore, the correlation of preoperative thrombocytosis with disease prognosis raises the possibility that marked serum levels may be linked to PLM in cervical cancer.

Because PLM is a major prognostic factor in managing SCC, it would be of great value for clinicians to pinpoint the situation of pelvic lymph nodes before surgery, if possible. In this study, we attempted to identify the factors related to PLM and to determine the significance of preoperative SCC-Ag and thrombocytosis in predicting PLM for SCC in FIGO stages IA-IIA.

\section{Materials and methods}

\subsection{Patients}

Seven hundred eighty-two patients with SCC who received diagnoses from January 2010 to October 2015 were enrolled in the primary cohort from three Chinese hospitals (Nanfang Hospital, Southern Medical University; Tongji Hospital, Huazhong University of Science and Technology; Xiangyang Central Hospital, Hubei University of Arts and Science). Four hundred seven patients were included in the validation cohort from 2015 to 2017 in another institution (Sun Yat-sen University Cancer Center, Sun Yat-sen University). Their data were retrospectively collected and analyzed. All patients were definitively diagnosed by two pathologists after a second examination of specimen slides. The 1189 patients with SCC in FIGO stage IA-IIA were undergoing primary radical hysterectomy with pelvic lymphadenectomy. This study was approved by the Ethics Committee of Nanfang Hospital/The First School of Clinical Medicine, Southern Medical University. Because of the retrospective study design, informed consent could not be obtained from each patients. Instead of obtaining informed consent from each patient, we posted a notice about the study design and contact information at a public location in Nanfang hospital.

\subsection{Methods}

The preoperative SCC-Ag and platelet levels of these patients, with diagnosis of early-stage SCC (stage IA to IIA), were examined through the following analyzers. The SCC-Ag level was measured before surgery without treatment with immunoradiometric assay (Imx, Abbott Diagnostics, Abbott Park, IL, USA) equipment. Patients were classified into two groups: a thrombocytosis group and a normal group. The thrombocytosis group was defined as having a platelet count greater than $300 \times$ $10^{9} / \mathrm{L}$ prior to primary treatment, and the platelet count of the normal group was in the range of $100 \sim$ $300 \times 10^{9} / \mathrm{L}$.

Data were analyzed with SPSS version 19.0. SCC-Ag levels and PLM status were evaluated through the receiver-operating characteristic (ROC) method [12] in the thrombocytosis group and the normal group. The best cutoff value was determined by maximization of the sum of the sensitivity and specificity. Univariate analysis and multivariate logistic regressions were used to evaluate the relationship between the selected risk factors and the PLM present for SCC in FIGO stage IA-IIA.

\section{Results}

\subsection{Clinical features of primary cohort and validation cohort}

The 782 patients of the primary cohort and 407 patients of the validation cohort with SCC in FIGO stage IA-IIA were retrospectively enrolled in the study and analyzed. Six hundred sixty-four patients $(84.9 \%)$ were PLM negative, whereas 118 (15.1\%) were PLM positive in the primary cohort; 309 patients (75.9\%) were PLM negative, whereas 98 (24.1\%) were PLM positive in validation cohort. Thrombocytosis was present in $15.6 \%(122 / 782)$ of patients in the primary cohort and $25.3 \%(103 / 407)$ of patients in the validation cohort. The mean level of SCC-Ag was 4.72 
$\mathrm{ng} / \mathrm{ml}$ for the primary cohort and $5.03 \mathrm{ng} / \mathrm{ml}$ for the validation cohort. Similar clinical characteristics were observed in both cohorts (Table 1).

Table 1. Clinical characteristics of patients with SCC in early FIGO stage

\begin{tabular}{|c|c|c|c|c|}
\hline Characteristic & $\begin{array}{l}\text { All patients } \\
\text { No. }(\%)\end{array}$ & $\begin{array}{l}\text { Primary cohort } \\
\text { No. }(\%)\end{array}$ & $\begin{array}{l}\text { Validation cohort } \\
\text { No. }(\%)\end{array}$ & P-value \\
\hline Total & 1189 & 782 & 407 & \\
\hline $\begin{array}{l}\text { Preoperative SCC-Ag } \\
\text { (ng/mL, mean) }\end{array}$ & 4.83 & 4.72 & 5.03 & 0.59 \\
\hline Age (year) & & & & $<0.05$ \\
\hline$\leq 50$ & 704 (59.2) & $503(64.3)$ & $201(49.4)$ & \\
\hline$>50$ & $485(40.8)$ & $279(35.7)$ & $206(50.6)$ & \\
\hline PLT level $\left(\times 10^{\wedge} 9 / \mathrm{L}\right)$ & & & & $<0.05$ \\
\hline$\leq 300$ & $964(81.1)$ & $660(84.4)$ & $304(74.7)$ & \\
\hline$>300$ & $225(18.9)$ & $122(15.6)$ & $103(25.3)$ & \\
\hline FIGO stage & & & & $<0.05$ \\
\hline IA-IB & 777 (65.3) & $527(67.4)$ & $250(61.4)$ & \\
\hline IIA & $412(34.7)$ & $255(32.6)$ & 157 (38.6) & \\
\hline Grade & & & & 0.35 \\
\hline Good or moderate & $916(77.0)$ & $596(76.2)$ & $320(78.6)$ & \\
\hline Poor & $273(23.0)$ & $186(23.8)$ & $87(21.4)$ & \\
\hline PI & & & & $<0.05$ \\
\hline Negative & $1151(96.8)$ & 771 (98.6) & $380(93.4)$ & \\
\hline Positive & $38(3.2)$ & $11(1.4)$ & $27(6.6)$ & \\
\hline LVSI & & & & $<0.05$ \\
\hline Negative & 945 (79.5) & $731(93.5)$ & $214(52.6)$ & \\
\hline Positive & $244(20.5)$ & $51(6.5)$ & $193(47.4)$ & \\
\hline DSI & & & & $<0.05$ \\
\hline$\leq 2 / 3$ & $676(56.9)$ & $499(63.8)$ & $177(43.5)$ & \\
\hline$>2 / 3$ & $513(43.1)$ & $283(36.2)$ & $230(56.5)$ & \\
\hline Tumor size & & & & 0.19 \\
\hline$\leq 4 \mathrm{~cm}$ & 993 (83.5) & $661(84.5)$ & 332 (81.6) & \\
\hline$>4 \mathrm{~cm}$ & $196(16.5)$ & $121(15.5)$ & $75(18.4)$ & \\
\hline PLM & & & & $<0.05$ \\
\hline Negative & $973(81.8)$ & $664(84.9)$ & 309 (75.9) & \\
\hline Positive & $216(18.2)$ & $118(15.1)$ & $98(24.1)$ & \\
\hline
\end{tabular}

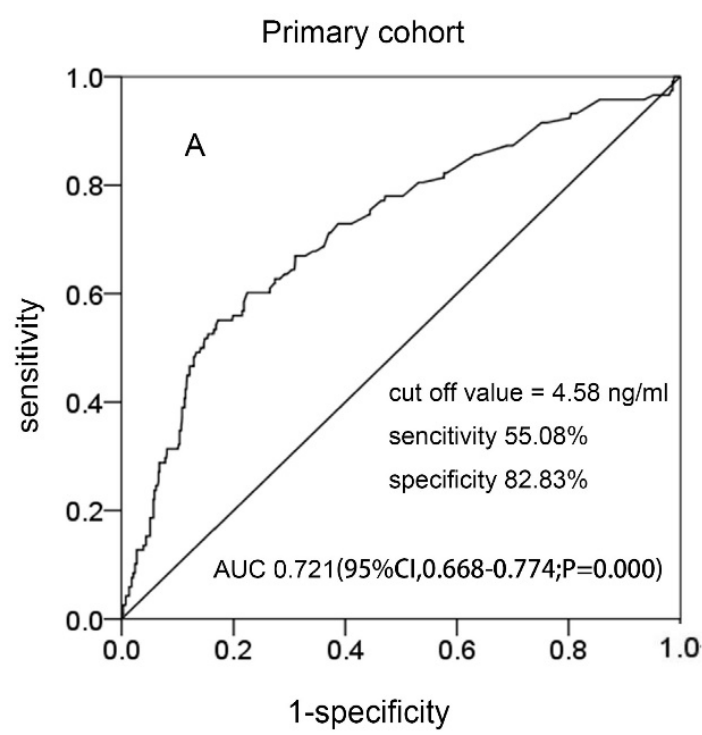

\subsection{Diagnostic value of SCC-Ag for PLM with thrombocytosis}

In the two cohorts, Figure 1 shows that the best cutoff value of preoperative SCC-Ag levels for PLM in the primary cohort and the validation cohort was 4.58 $\mathrm{ng} / \mathrm{mL}$ (area under the curve [AUC], 0.721; 95\% confidence interval [CI] 0.668-0.774; sensitivity, $55.08 \%$; specificity, $82.03 \%$ ) and $1.55 \mathrm{ng} / \mathrm{mL}$ (AUC, 0.670; 95\% CI 0.608-0.731; sensitivity, 75.51\%; specificity, $58.58 \%$ ), respectively.

Patients were classified into two groups: the thrombocytosis group and the normal group. In the primary cohort, the best cutoff value of preoperative SCC-Ag levels at primary treatment for PLM in the thrombocytosis group and the normal group were $3.26 \mathrm{ng} / \mathrm{mL}$ (AUC, 0.754; 95\% CI 0.649-0.860; sensitivity, 73.08\%; specificity, $72.92 \%)$ and 4.58 ng/mL (AUC, 0.707; 95\% CI 0.608-0.731; sensitivity, $54.44 \%$; specificity, $83.15 \%$ ) (Fig. $2 \mathrm{~A}$ and Fig. 2B). In the validation cohort, the best cutoff value of preoperative SCC-Ag levels at primary treatment for PLM in the thrombocytosis group and the normal group were $1.55 \mathrm{ng} / \mathrm{mL}$ (AUC, 0.705; 95\% CI 0.588-0.822; sensitivity, $79.31 \%$; specificity, $55.41 \%$ ) and $1.75 \mathrm{ng} / \mathrm{mL}$ (AUC, 0.655; 95\% CI 0.583-0.728; sensitivity, 69.57\%; specificity, 64.26\%) (Fig. 2C and Fig. 2D).

The value of SCC-Ag to discriminate between the thrombocytosis group and the normal group was analyzed by ROC curves (Table 2). By comparing the sensitivity, specificity, and AUC in the two groups, we found that the combination of SCC-Ag above 3.26 $\mathrm{ng} / \mathrm{mL}$ and thrombocytosis was more sensitive.

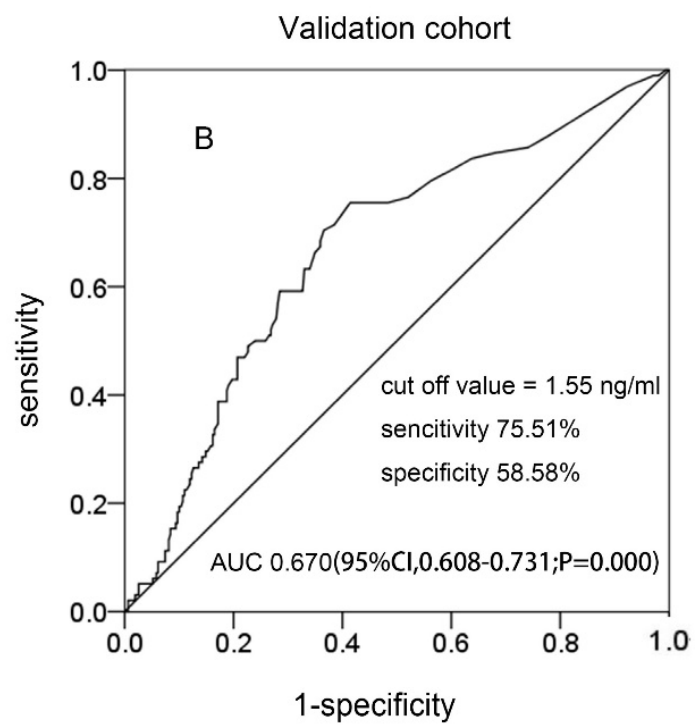

Figure 1. The ROC curve of preoperative SCC-Ag for PLM in different cohorts: A, the ROC curve of preoperative SCC-Ag level to PLM in primary cohort; B, the ROC curve of preoperative SCC-Ag level for PLM in validation cohort. 

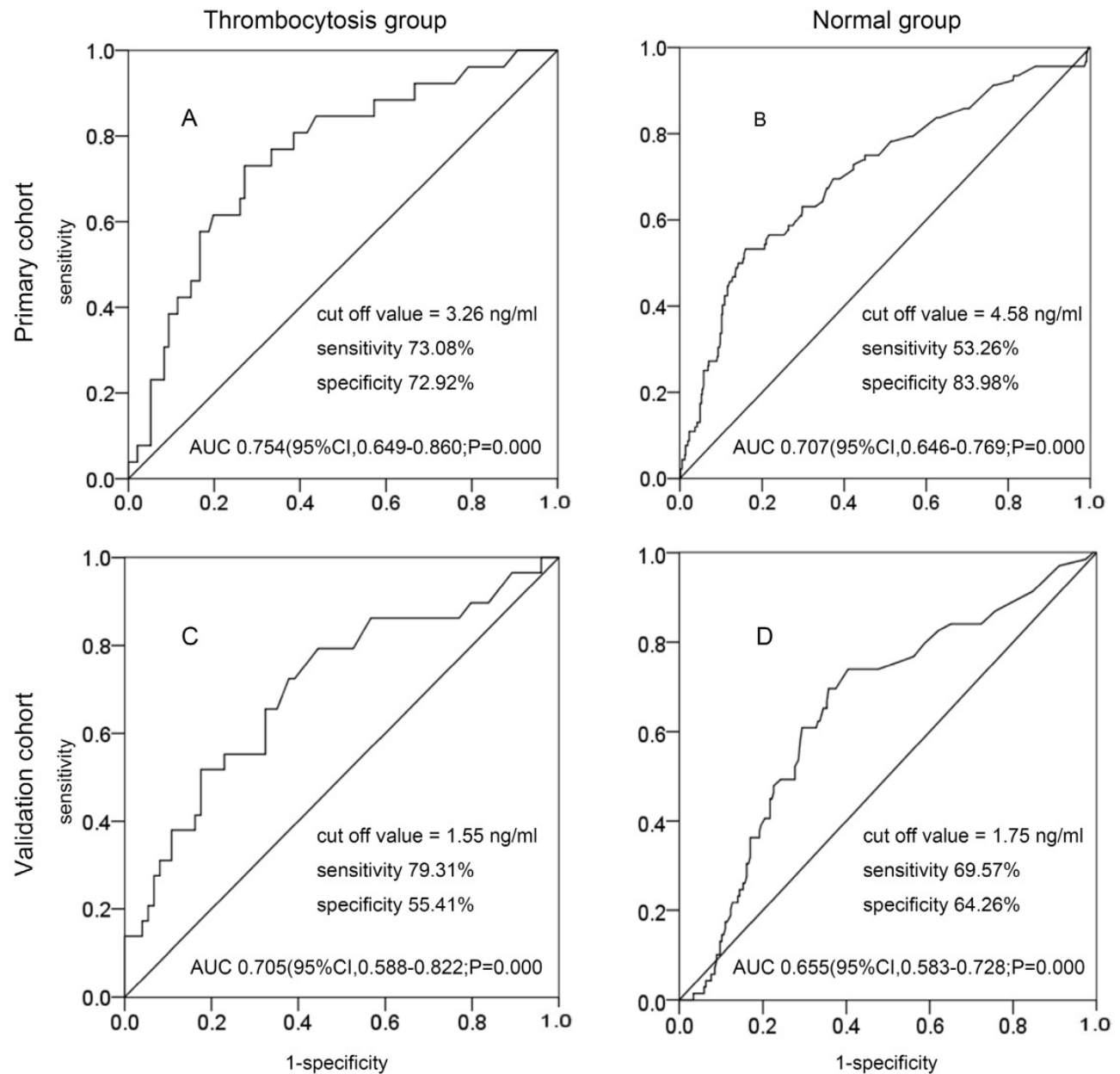

Figure 2. The ROC curve of preoperative SCC-Ag for PLM in primary cohort and validation cohort: A, the ROC curve of preoperative SCC-Ag level to PLM in the thrombocytosis group of primary cohort (PLT level $>300 \times 10 \% / \mathrm{L}$ ); B, the ROC curve of preoperative SCC-Ag level for PLM in the normal group of primary cohort (PLT level $\left.\leq 300 \times 10^{9} / \mathrm{L}\right)$; C, the ROC curve of preoperative SCC-Ag level to PLM in the thrombocytosis group of validation cohort (PLT level >300 $\left.\times 10^{9} / \mathrm{L}\right) ; \mathrm{D}$, the ROC curve of preoperative SCC-Ag level for PLM in the normal group of validation cohort (PLT level $\leq 300 \times 10^{9} / \mathrm{L}$ ).

Table 2. Diagnostic value of SCC-Ag for PLM involvement in the thrombocytosis group and the normal group

\begin{tabular}{llllll}
\hline Cohort & ROC & \multicolumn{2}{l}{$\begin{array}{l}\text { Thrombocytosis } \\
\text { group }\end{array}$} & \multicolumn{2}{l}{ Normal group } \\
\cline { 3 - 6 } & & value & $95 \% \mathrm{CI}$ & value & $95 \% \mathrm{CI}$ \\
\hline $\begin{array}{l}\text { primary cohor } \\
(\mathrm{n}=782)\end{array}$ & AUCROC & 0.75 & $0.65-0.86$ & 0.71 & $0.65-0.77$ \\
& $\begin{array}{l}\text { SCC-Ag, cutoff } \\
\text { (ng/ml) }\end{array}$ & 3.26 & & 4.58 & \\
& Sensitivity (\%) & 73.08 & $52.20-88.40$ & 53.26 & $42.60-63.70$ \\
& Specificity (\%) & 72.92 & $62.90-81.50$ & 83.98 & $80.70-86.90$ \\
& PPV (\%) & 42.22 & $27.50-58.00$ & 35.00 & $27.10-43.50$ \\
& NPV (\%) & 90.91 & $82.20-96.30$ & 91.73 & $89.00-94.00$ \\
& LR+ & 2.70 & $2.10-3.50$ & 3.32 & $2.70-4.00$ \\
Validation & LR- & 0.37 & $0.20-0.80$ & 0.56 & $0.40-0.70$ \\
cohort & AUCROC & 0.71 & $0.59-0.82$ & 0.66 & $0.58-0.73$ \\
$(\mathrm{n}=407)$ & SCC-Ag, cutoff & 1.55 & & & \\
& (ng/ml) & & & 1.75 & \\
& Sensitivity (\%) & 79.31 & $60.30-92.00$ & 69.57 & $57.30-80.10$ \\
& Specificity (\%) & 55.41 & $43.40-67.00$ & 64.26 & $57.80-70.40$ \\
& PPV (\%) & 41.07 & $28.10-55.00$ & 36.36 & $28.10-45.20$ \\
& NPV (\%) & 87.23 & $74.10-95.20$ & 87.79 & $81.90-92.30$ \\
& LR+ & 1.78 & $1.30-2.30$ & 1.95 & $1.60-2.30$ \\
& LR- & 0.37 & $0.20-0.80$ & 0.47 & $0.30-0.70$ \\
\hline
\end{tabular}

SCC-Ag, squamous cell carcinoma antigen; PLM, pelvic lymphatic metastasis; PPV, positive predictive value; NPV, negative predictive value; LR+, positive likelihood ratio (sensitivity/1-specificity); LR-, negative likelihood ratio

(1-sensitivity/specificity); CI, confidence interval; ROC, receiver operating

characteristic curve; AUROC, area under receiver operating characteristic curve

\subsection{Univariate and multivariate analysis of variables to PLM}

Univariate and multivariate logistic analyses of clinicopathological factors of PLM are shown in Table 3 and Table 4 . The following factors have significant effects on PLM through univariate analysis: preoperative SCC-Ag $>3.26 \mathrm{ng} / \mathrm{mL} \quad(\mathrm{p}<0.05)$, thrombocytosis $(\mathrm{p}<0.05)$, advanced FIGO stage $(\mathrm{p}<0.05)$, parametrial involvement $(\mathrm{PI})(+)(\mathrm{p}<0.05)$, lymphovascular space involvement (LVSI) $(+)$ $(p<0.05)$, the depth of stromal invasion (DSI) $>2 / 3$ $(\mathrm{p}<0.05)$, and tumor size $>4 \mathrm{~cm}(\mathrm{p}<0.05)$ in the primary cohort, and preoperative SCC-Ag $>3.26$ $\mathrm{ng} / \mathrm{mL}(\mathrm{p}<0.05)$, advanced FIGO stage $(\mathrm{p}<0.05), \mathrm{PI}(+)$ $(p<0.05)$, LVSI $(+)(p<0.05)$, DSI $>2 / 3(p<0.05)$, and tumor size $>4 \mathrm{~cm} \quad(\mathrm{p}<0.05)$ in the validation cohort. Through multivariate logistic regression, we found that preoperative SCC-Ag $>3.26 \mathrm{ng} / \mathrm{mL}(\mathrm{p}<0.05)$, and LVSI $(+)(p<0.05)$ increase the likelihood of positive PLM in the two cohorts (Table 4). 
Table 3. PLM in univariate analysis for patients with SCC in early FIGO stage

\begin{tabular}{|c|c|c|c|c|c|c|}
\hline \multirow[t]{2}{*}{ Characteristic } & \multicolumn{3}{|c|}{ Primary cohort(n = 782) } & \multicolumn{3}{|c|}{ Validation cohort(n = 407) } \\
\hline & $\begin{array}{l}\operatorname{PLM}(-) \\
(\%)\end{array}$ & $\begin{array}{l}\operatorname{PLM}(+) \\
(\%)\end{array}$ & P-value & $\begin{array}{l}\text { PLM(-) } \\
(\%)\end{array}$ & $\begin{array}{l}\operatorname{PLM}(+) \\
(\%)\end{array}$ & P-value \\
\hline $\begin{array}{l}\text { Preoperative } \\
\text { SCC-Ag }\end{array}$ & & & $<0.05$ & & & $<0.05$ \\
\hline$\leq 3.26$ & $514(77.4)$ & $47(39.8)$ & & $222(71.8)$ & $42(42.9)$ & \\
\hline$>3.26$ & $150(22.6)$ & $71(60.2)$ & & $87(28.2)$ & $56(57.1)$ & \\
\hline Age(year) & & & 0.20 & & & 0.43 \\
\hline$\leq 50$ & $421(63.4)$ & $82(69.5)$ & & $156(50.5)$ & $45(45.9)$ & \\
\hline$>50$ & $243(36.6)$ & $36(30.5)$ & & $153(49.5)$ & $53(54.1)$ & \\
\hline $\begin{array}{l}\text { PLT level } \\
\left(\times 10^{\wedge} 9 / \mathrm{L}\right)\end{array}$ & & & $<0.05$ & & & 0.26 \\
\hline$\leq 300$ & $568(85.5)$ & $92(78.0)$ & & $235(76.1)$ & $69(70.4)$ & \\
\hline$>300$ & $96(14.5)$ & $26(22.0)$ & & $74(23.9)$ & $29(29.6)$ & \\
\hline FIGO stage & & & $<0.05$ & & & $<0.05$ \\
\hline IA-IB & $462(69.6)$ & $65(55.1)$ & & $200(64.7)$ & $50(51.0)$ & \\
\hline IIA & $202(30.4)$ & $53(44.9)$ & & $109(35.3)$ & $48(49.0)$ & \\
\hline Grade & & & 0.80 & & & 0.79 \\
\hline Good or moderate & $505(76.1)$ & $91(77.1)$ & & $242(78.3)$ & $78(79.6)$ & \\
\hline Poor & $159(23.9)$ & $27(22.9)$ & & $67(21.7)$ & $20(20.4)$ & \\
\hline PI & & & $<0.05$ & & & $<0.05$ \\
\hline Negative & $660(99.4)$ & 111(94.1) & & $300(97.1)$ & 80 (81.6) & \\
\hline Positive & $4(0.6)$ & $7(5.9)$ & & $9(2.9)$ & 18 (18.4) & \\
\hline LVSI & & & $<0.05$ & & & $<0.05$ \\
\hline Negative & 634 (95.5) & $97(82.2)$ & & 195 (63.1) & 19 (19.4) & \\
\hline Positive & $30(4.5)$ & 21(17.8) & & $114(36.9)$ & 79 (80.6) & \\
\hline DSI & & & $<0.05$ & & & $<0.05$ \\
\hline$\leq 2 / 3$ & $446(67.2)$ & $53(44.9)$ & & $143(46.3)$ & $34(34.7)$ & \\
\hline$>2 / 3$ & $218(32.8)$ & $65(55.1)$ & & $166(53.7)$ & $64(65.3)$ & \\
\hline Tumor size & & & $<0.05$ & & & $<0.05$ \\
\hline$\leq 4 \mathrm{~cm}$ & $570(85.8)$ & 91(77.1) & & $264(85.4)$ & $68(69.4)$ & \\
\hline$>4 \mathrm{~cm}$ & $94(14.2)$ & $27(22.9)$ & & 45 (14.6) & 30 (30.6) & \\
\hline
\end{tabular}

Table 4. Multivariable logistic regression for the prediction of PLM

\begin{tabular}{|c|c|c|c|c|c|c|}
\hline \multirow[t]{2}{*}{ Variable } & \multicolumn{3}{|c|}{ Primary cohort $(\mathrm{n}=782)$} & \multicolumn{3}{|c|}{ Validation cohort $(n=407)$} \\
\hline & $\begin{array}{l}\text { Odds } \\
\text { ratio }\end{array}$ & $95 \% \mathrm{CI}$ & P-value & $\begin{array}{l}\text { Odds } \\
\text { ratio }\end{array}$ & $95 \% \mathrm{CI}$ & P-value \\
\hline Age >50 (year) & 0.703 & $0.434-1.139$ & 0.15 & 1.074 & $0.620-1.860$ & 0.80 \\
\hline $\begin{array}{l}\text { Preoperative SCC-Ag } \\
>3.26 \mathrm{ng} / \mathrm{mL}\end{array}$ & 4.106 & $2.589-6.511$ & $<0.05$ & 3.022 & $1.720-5.309$ & $<0.05$ \\
\hline Thrombocytosis & 1.311 & $0.757-2.269$ & 0.33 & 1.135 & $0.623-2.067$ & 0.68 \\
\hline $\begin{array}{l}\text { FIGO stage (IIA vs. } \\
\text { IA-IB) }\end{array}$ & 1.648 & $1.048-2.593$ & $<0.05$ & 1.362 & $0.770-2.410$ & 0.29 \\
\hline $\begin{array}{l}\text { Grade (poor vs. } \\
\text { others) }\end{array}$ & 0.817 & $0.488-1.366$ & 0.44 & 1.022 & $0.532-1.964$ & 0.95 \\
\hline PI $(+)$ & 2.551 & $0.626-10.390$ & 0.19 & 3.345 & $1.305-8.577$ & $<0.05$ \\
\hline LVSI (+) & 5.031 & $2.531-10.002$ & $<0.05$ & 7.132 & $3.928-12.949$ & $<0.05$ \\
\hline DSI $>2 / 3$ & 1.525 & $0.968-2.404$ & 0.07 & 0.763 & $0.427-1.364$ & 0.36 \\
\hline Tumor size $>4 \mathrm{~cm}$ & 1.102 & $0.639-1.901$ & 0.73 & 1.554 & $0.807-2.992$ & 0.19 \\
\hline
\end{tabular}

SCC-Ag, squamous cell carcinoma antigen; FIGO, International Federation of Gynecology and Obstetrics; PI, parametrial involvement; LVSI, lymphovascular space involvement; DSI, depth of stromal invasion; PLM, pelvic lymphatic metastasis

\section{Discussion}

Lymphatic metastasis is the main factor affecting the outcome of cervical carcinoma in early-stage SCC [13-15]. On one hand, micro-metastases are identifiable in histologically negative PLM in $15 \%$ of early-stage cervical cancer patients, a frequency that approximates the recurrence rate of patients with negative nodes [16-17], on the other hand, lower body lymphedema is a significant cause of morbidity following the pelvic lymph node dissection that strongly impacts patients' quality of life (QoL) [18]. It is, therefore, important to identify positive PLM in patients with SCC in an early FIGO stage. However, there are no independent indicators to predict PLM in cervical cancer before initial treatment. It is well known that the serum level of SCC-Ag is well correlated with clinical stage or tumor spread [13]. It has also been shown to be associated with PLM in SCC before primary treatment [19-22], but the predicting value of preoperative SCC-Ag is controversial. In this paper, as shown in Table 3 and Table 4, preoperative SCC-Ag > $3.26 \mathrm{ng} / \mathrm{mL}$ increases the likelihood of positive PLM, which might be useful for diagnosing PLM in SCC. Patients with higher pretreatment SCC-Ag levels are prone to show positive PLM (Table 4). Nevertheless, preoperative SCC-Ag was insufficiently reliable to diagnose PLM because of its low sensitivity (Figure 1), which is in accordance with the previous study $[8,21]$.

An acceptable sensitivity for diagnosing PLM may be obtained by combining SCC-Ag with other markers. The association between thrombocytosis and malignancies has been well demonstrated [23-26]. The data obtained by the previous and present studies suggest that thrombocytosis reflects a more aggressive tumor biology. Andersen et al [27] showed that platelet count may play an important role in diagnosis and post-diagnosis control of gynecological cancer. Cheng et al [28] also identified that cervical cancer patients with pretreatment have an elevated platelet count and are prone to suffer positive PLM. To date, there have been no studies on combination assay of preoperative SCC-Ag and thrombocytosis in predicting PLM in early-stage SCC. In this paper, pretreatment thrombocytosis was also related to PLM (Table 3). Moreover, the combination of preoperative SCC-Ag and thrombocytosis seems to improve the sensitivity of SCC-Ag for diagnosing PLM before initial treatment (Table 2). The differences in sensitivity of the best cutoff value of the normal group and the thrombocytosis group probably reflect the effect of tumor metastases on SCC-Ag levels in the latter. Because of tumor progression in patients with thrombocytosis, an elevated marker is more likely to reflect the presence of PLM in the thrombocytosis group than in the normal group.

Although we can combine SCC-Ag and thrombocytosis to predict PLM in early-stage SCC, the mechanisms underlying this observation are not fully defined. Murakami et al [29] suggested that SCC-Ag may be involved in metastasis through changing E-cadherin expression. Moreover, the heterogeneous 
pattern of SCC-Ag and E-cadherin in a primary lesion is tightly associated with the high incidence of lymph node metastasis in SCC [30]. The loss of E-cadherin plays an important role in the progression of cancer cells and is associated with their metastasis. Mounting evidence reveals that the down-regulation of E-cadherin results in less intercellular adhesion and less cell polarity [31, 32]. Consequently, epithelial cells become mesenchymal stem cells, which is one hallmark of epithelial-mesenchymal transition (EMT) [33].

The mechanisms of the relationship between thrombocytosis and PLM are still unknown [34, 35]. Experimental evidence shows that angiogenesis is crucial in tumor proliferation and metastasis; all growth factors; and cytokines such as VEGF, PDGF, FGF, TGF $\beta$, and IL-6. They contribute to inducing EMT in the microenvironment of tumor cells and stimulating the angiogenic process [36,37]. Stone et al [9] reported that thrombocytosis might be a paraneoplastic syndrome that expresses itself through tumor-derived IL-6, which activates thrombopoiesis and then results in thrombocytosis and tumor progression. Furthermore, the latest evidence shows that incubating platelets with cancer cell lines will strengthen cell proliferation [38]. Recently, a published study on breast cancer proposed that platelet-rich plasma mimics the network of fibrin bundles in breast cancer environment. Therefore, it promotes the selection of cells with the most potential for malignancy, activates the EMT process, and enhances proteolytic activity [39].

Because pretreatment SCC-Ag and thrombocytosis seemed to be related to the EMT process independently, and platelet-rich plasma was likely to select the most potential malignancy cell, these observations led us to construct an index that includes two tumor markers. As shown in Figure 2, SCC-Ag could predict PLM more sensitively in the thrombocytosis group than in the normal group. The current study demonstrated the usefulness of a combination assay of serum SCC-Ag and thrombocytosis in predicting PLM based on the data of patients who had undergone radical hysterectomy and pelvic lymphadenectomy. The markers that could identify the subgroup of PLM-positive patients would be useful for making decisions before primary treatment.

Our study provides evidence that preoperative SCC-Ag may play a role in the pretreatment evaluation in early FIGO stage SCC for PLM, and combining SCC-Ag and thrombocytosis can improve the sensitivity of SCC-Ag for predicting PLM. Because SCC-Ag and platelet count measurement are available and well standardized for every clinical patient, they may be used as a convenient and useful serum biomarker to provide conventional clinicopathological variables to help clinicians estimate positive PLM for SCC before primary treatment.

One limitation of the present study was mainly related to its retrospective nature and reliance on preexisting data collections for analysis. Given this limitation, future prospective studies are needed to determine the risk of SCC-Ag and thrombocytosis for PLM more accurately. Another limitation was that the potential selection bias could not be completely excluded due to the enrollment of only four institutions. A third limitation of this study was the small number of patients, and standardization of all clinical assays was difficult. However, we believe that the diagnostic value of SCC-Ag for PLM involvement with thrombocytosis could be of interest to clinicians.

\section{Acknowledgements}

This work was supported by the National Science Foundation of China (No. 81372781; 81672589; 81072132; 81302249); the National Key Research and Development Program of China (No. 2016 YFC1302901); and the Science and Technology Program of Shenzhen (No. JCYJ20160429161218745).

\section{Competing Interests}

The authors have declared that no competing interest exists.

\section{References}

1. Lim S, Cho K, Lee $\mathrm{S}$, et al. Effect of number of retrieved lymph nodes on prognosis in FIGO stage IB-IIA cervical cancer patients treated with primary radical surgery. J Obstet Gynaecol Res. 2017; 43:211-219.

2. Wang W, Jia HL, Huang JM, et al. Identification of biomarkers for lymph node metastasis in early-stage cervical cancer by tissue-based proteomics. Br J Cancer. 2014; 110:1748-1758.

3. Wu ES, Jeronimo J, Feldman S. Barriers and Challenges to Treatment Alternatives for Early-Stage Cervical Cancer in Lower-Resource Settings. J Glob Oncol. 2017; 3:572-582.

4. Kamura T, Tsukamoto N, Tsuruchi N, et al. Multivariate analysis of the histopathologic prognostic factors of cervical cancer in patients undergoing radical hysterectomy. Cancer. 1992; 69:181-186.

5. Zigras T, Lennox G, Willows $\mathrm{K}$, et al. Early Cervical Cancer: Current Dilemmas of Staging and Surgery. Curr Oncol Rep. 2017;19:51-52.

6. Salvatici M, Achilarre MT, Sandri MT, et al. Squamous cell carcinoma antigen (SCC-Ag) during follow-up of cervical cancer patients: Role in the early diagnosis of recurrence. Gynecol Oncol. 2016; 142:115-119.

7. Ryu HK, Baek JS, Kang WD, et al. The prognostic value of squamous cell carcinoma antigen for predicting tumor recurrence in cervical squamous cell carcinoma patients. Obstet Gynecol Sci. 2015; 58:368-376.

8. Gaarenstroom KN, Kenter GG, Bonfrer JM, et al. Can initial serum cyfra 21-1, SCC antigen, and TPA levels in squamous cell cervical cancer predict lymph node metastases or prognosis? Gynecol Oncol. 2000; 77:164-170.

9. Stone RL, Nick AM, McNeish IA, et al. Paraneoplastic thrombocytosis in ovarian cancer. N Engl J Med. 2012; 366:610-618.

10. Takahashi R, Mabuchi S, Kuroda H, et al. The Significance of Pretreatment Thrombocytosis and Its Association With Neutrophilia in Patients With Surgically Treated Endometrial Cancer. Int J Gynecol Cancer. 2017; 27:1399-1407.

11. Yu M, Liu L, Zhang BL, et al. Pretreatment thrombocytosis as a prognostic factor in women with gynecologic malignancies: a meta-analysis. Asian Pac J Cancer Prev. 2012; 13:6077-6081.

12. Zweig MH, Campbell G. Receiver-operating characteristic (ROC) plots: a fundamental evaluation tool in clinical medicine. Clin Chem. 1993; 39:561-577.

13. Lecuru F, Mathevet $P$, Querleu D, et al. Bilateral negative sentinel nodes accurately predict absence of lymph node metastasis in early cervical cancer: results of the SENTICOL study. J Clin Oncol. 2011; 29:1686-1691. 
14. Paredes P, Vidal-Sicart S, Campos F, et al. Role of ICG- $(99 \mathrm{~m})$ Tc-nanocolloid for sentinel lymph node detection in cervical cancer: a pilot study. Eur J Nucl Med Mol Imaging. 2017; 44:1853-1861.

15. Lee YJ, Kim DY, Lee SW, et al. A postoperative scoring system for distant recurrence in node-positive cervical cancer patients after radical hysterectomy and pelvic lymph node dissection with para-aortic lymph node sampling or dissection. Gynecol Oncol. 2017;144:536-540.

16. Cibula D, Zikan M, Slama J, et al. Risk of micrometastases in non-sentinel pelvic lymph nodes in cervical cancer. Gynecol Oncol. 2016; 143:83-86.

17. Lentz SE, Muderspach LI, Felix JC, et al. Identification of micrometastases in histologically negative lymph nodes of early-stage cervical cancer patients. Obstet Gynecol. 2004; 103:1204-1210.

18. Biglia N, Zanfagnin V, Daniele A, et al. Lower Body Lymphedema in Patients with Gynecologic Cancer. Anticancer Res. 2017; 37:4005-4015.

19. Dasari S, Wudayagiri R, Valluru L. Cervical cancer: Biomarkers for diagnosis and treatment. Clin Chim Acta. 2015; 445:7-11.

20. Iida M, Banno K, Yanokura M, et al. Candidate biomarkers for cervical cancer treatment: Potential for clinical practice (Review). Mol Clin Oncol, 2014; 2: 647-655.

21. van de Lande J, Davelaar EM, von Mensdorff-Pouilly S, et al. SCC-Ag, lymph node metastases and sentinel node procedure in early stage squamous cell cervical cancer. Gynecol Oncol. 2009; 112:119-125.

22. Zhou Z, Li W, Zhang F, et al. The value of squamous cell carcinoma antigen (SCCa) to determine the lymph nodal metastasis in cervical cancer: A meta-analysis and literature review. PLoS One. 2017; 11:e0186165.

23. Rao AK, Rao DA. Platelets signal and tumors take off. Blood. 2012; 120:4667-4668

24. Wang L, Jia J, Lin L, et al. Predictive value of hematological markers of systemic inflammation for managing cervical cancer. Oncotarget, 2017; 8:44824-44832.

25. Bordon Y. Tumour immunology: Platelets - a new target in cancer immunotherapy? Nat Rev Immunol. 2017; 17:348-349.

26. Haemmerle M, Taylor ML, Gutschner T, et al. Platelets reduce anoikis and promote metastasis by activating YAP1 signaling. Nat Commun. 2017; 21:310-311.

27. Andersen CL, Eskelund CW, Siersma VD, et al. Is thrombocytosis a valid indicator of advanced stage and high mortality of gynecological cancer? Gynecol Oncol. 2015; 139:312-318.

28. Cheng J, Zeng Z, Ye Q, et al. The association of pretreatment thrombocytosis with prognosis and clinicopathological significance in cervical cancer: a systematic review and meta-analysis. Oncotarget. 2017; 8:24327-24336.

29. Murakami A, Nakagawa T, Fukushima C, et al. Relationship between decreased expression of squamous cell carcinoma antigen 2 and E-cadherin in primary cervical cancer lesions and lymph node metastasis. Oncol Rep. 2008; 19:99-104.

30. Schipper JH, Frixen UH, Behrens J, et al. E-cadherin expression in squamous cell carcinomas of head and neck: inverse correlation with tumor dedifferentiation and lymph node metastasis. Cancer Res. 1991; 51:6328-6337.

31. Lv X, Hou M, Duan X. Correlation analysis between the parameters of contrast-enhanced ultrasonography in evaluating cervical cancer metastasis and expression of E-cadherin. Oncol Lett. 2017; 14:4641-4646.

32. Jiang $\mathrm{Y}$, Ren $\mathrm{W}$, Wang $\mathrm{W}$, et al. Inhibitor of $\beta$-catenin and TCF (ICAT) promotes cervical cancer growth and metastasis by disrupting E-cadherin/ $\beta$-catenin complex. Oncol Rep. 2017; 38:2597-2606.

33. Scarpa E, Szabo A, Bibonne A, et al. Cadherin Switch during EMT in Neural Crest Cells Leads to Contact Inhibition of Locomotion via Repolarization of Forces. Dev Cell. 2015; 34:421-434

34. Menczer J. Preoperative elevated platelet count and thrombocytosis in gynecologic malignancies. Arch Gynecol Obstet. 2017; 295:9-15.

35. De Jonge E T, Viljoen E, Lindeque B G, et al. The prognostic significance of p53, mdm2, c-erbB-2, cathepsin D, and thrombocytosis in stage IB cervical cancer treated by primary radical hysterectomy. Int J Gynecol Cancer, 1999; 9:198-205.

36. Jain S, Harris J, Ware J. Platelets: linking hemostasis and cancer. Arterioscler Thromb Vasc Biol. 2010; 30:2362-2367.

37. Sharma D, Singh G. Thrombocytosis in gynecological cancers. J Cancer Res Ther. 2017; 13: 193-197

38. Cho MS, Bottsford-Miller J, Vasquez HG, et al. Platelets increase the proliferation of ovarian cancer cells. Blood. 2012; 120:4869-4872.

39. Davis FM, Azimi I, Faville RA, et al. Induction of epithelial-mesenchymal transition (EMT) in breast cancer cells is calcium signal dependent. Oncogene. $2014 ; 33: 2307-2316$ 